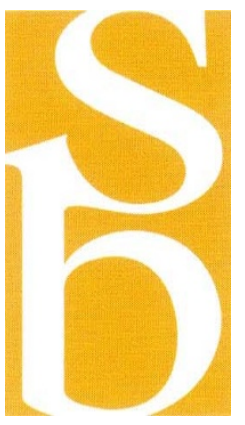

make up multicellular plants and animals. Indeed, there are suggestions that the 2.5 billion years it took for multicellular organisms to appear may have been related to the need to develop the complex signalling mechanisms that allowed intercellular communication and the coordination of cellular behaviour. A mere twenty years ago the nature of these cellular information highways was, for the most part, a black box. Since then a vast effort has been directed towards shedding light on this area of biology. The importance of the endeavour has now been recognized by the award of the 1994 Nobel Prize for Medicine to the discoverers of G-protein-mediated signal transduction; Martin Rodbell, recently retired from the National Institute of Environmental Health Sciences, North Carolina, and Alfred G. Gilman, of the University of Texas Southwestern.

Before the discovery of G-proteins it had been assumed that when a signal, such as a hormone, was bound by its cell-surface receptor, the activated receptor signalled directly to the effector in the cytoplasm. This view was dramatically revised in the early 1970s when Rodbell, then working at the National Institute of Health in Bethesda, demonstrated that a third component, a transducer, passed the incoming signal from receptor to effector. But it was not until 1980 that Gilman and colleagues, then at the University of Virginia in Charlottesville (and independently, Thomas Pfeufer, University of Wurzburg, Germany) managed to isolate and characterize the transducer, christening the molecule a

\section{intracellular cation}

G-protein because of its requirement for GTP. Indeed, the nature of the interaction between the rod-cell G-protein transducin and its cytoplasmic effector is explored in a paper from Margolskee and colleagues in this issue'.

\section{G-proteins}

There are three characterized classes of cellsurface receptor proteins: those linked to ion channels, enzymes and G-proteins. The last group of receptors, which typically have a seven-helix transmembrane structure, is by far the largest, its members being numbered in the hundreds. Cellular responses to an extensive range of stimuli - light, smell, taste, hormones, pheromones, neurotransmitters and the like - are all mediated through G-protein-coupled receptors.

$\mathrm{G}$-proteins are heterotrimers, made from $\alpha, \beta$ and $\gamma$ subunits $\left(G_{\alpha \beta \gamma}\right)$; each heterotrimer is coupled to a specific receptor, relaying the signals from the activated receptor to effectors (enzymes or ion channels) that then generate one or more intracellular second messengers, such as cyclic nucleotide, $\mathrm{Ca}^{2+}$, inositol phosphates or diacylglycerol. The specificity is clearly important in maintaining the fidelity of the signal transduction process. The $\alpha$-subunit determines the specificity of the G-protein/ receptor interaction whereas interaction with downstream effectors may be specified by $\mathrm{G}_{\alpha}$ and/or $\mathrm{G}_{\beta \gamma}$ subunits ${ }^{2}$. The various combinations of $\alpha \beta \gamma$ heterotrimer subunits allow for both subtle regulation and complex feedback control of receptors and effectors.

Cloning, expression and mutational analysis of a number of G-proteins has advanced understanding of the intricacies of the interactions between G-proteins and receptors, G-proteins and effectors, the mechanism and regulation of GTP hydroly- 
sis, the regulation of the membrane attachment of G-protein components, and so on (for example, ref. 3 and references therein). Nonetheless, the problem of how this class of proteins function is far from solved. What, for example, is the molecular basis of the specificity of the G-proteins? The carboxy terminus of the $\alpha$-subunit of the G-protein is one of the major determinants of receptor recognition, while the cytoplasmic loops that link the helices of the seven transmembrane receptors are the main determinants of Gprotein interaction. Sequences adjacent to the C-terminus of the $\alpha$-subunit have been implicated in effector interactions.

In rod receptor cells of the vertebrate eye the light responsive receptor, rhodopsin, is coupled to its effector, the enzyme cGMPspecific phosphodiesterase (PDE), by the heterotrimeric G-protein transducin $\left(G_{t \alpha \beta \gamma}\right)$. Inactive $G_{t \alpha \beta \gamma} \cdot G D P$ binds to the photoactivated rhodopsin and releases GDP. GTP then binds to the $\alpha$-subunit of the receptor-bound transducin causing $\mathrm{G}_{\mathrm{t} \text { o }} \cdot$ GTP to separate from both the receptor and the $\beta \gamma$ heterodimer. The $G_{t \alpha} \cdot$ GTP complex then activates PDE by binding to that protein's inhibitory $\gamma$-subunit. Activation of the phosphodiesterase lowers the concentration of cGMP, which closes cGMP-gated cation channels and hyperpolarizes the retinal rod cell, thereby generating the nerve impulse. The intrinsic GTPase activity of the $\alpha$ subunit hydrolyses the bound GTP to GDP, inactivating $G_{t a}$, releasing the inhibitory PDE $\gamma$-subunit which in turn switches off the phosphodiesterase.

What is the structural basis of the $G_{t \alpha}$ specificity for the PDE $\gamma$ subunit? Margolskee and colleagues have addressed this question by using varients of a synthetic peptide from a region of $\mathrm{G}_{\mathrm{t} \alpha}$ which on its own is able to interact with and inhibit the PDE $\gamma$-subunit. By mutating individual residues in a short region of the $G_{t \alpha}$, and by using peptides from similar regions of other closely related G-protein $\alpha$-subunit, the authors are able to show that five amino acid residues are particularly important for PDE activation. Three of these residues are within helix $\alpha 4$ of the transducin $\alpha$-subunit and all have their side chains exposed on the same, solvent exposed, face of the transducin molecule suggesting that this helix defines the primary site of interaction between $\alpha_{t-\text { rod }}$ and PDE $\gamma$. That result is at odds with previous suggestions that the adjacent $\alpha 4 / \beta 6$ loop is the site of interaction $^{3,5}$. There are potential problems associated with using peptides to mimic the activity of much larger proteins, as discussed by Artemyve and $\mathrm{Hamm}^{4}$, nonetheless the solvent-exposed location of all five residues and modelling of the various substitutions of these residues argue against such artifacts.

While the authors have focused on one particular region involved in the interaction between G-protein and effector, the fact that this region is not seen to undergo a conformational change on exchange in the crystal structures of $\mathrm{G}_{\mathrm{t \alpha}} \cdot \mathrm{GTP} \gamma \mathrm{S}$ (ref. 5) and $\mathrm{G}_{\mathrm{t \alpha}} \cdot \mathrm{GDP}$ (ref. 6) suggests that there must be other sites of interaction between the two proteins. Clearly, the structure of the complex between $G_{t \alpha} \cdot G T P$ and PDE $\gamma$ would help resolve this question. But the demands of cell biologists do not stop there: what of the interactions between $G \alpha$ and $G \beta \gamma$ ? how does $G \alpha$ interact with receptor? what are the parameters determining GTP hydrolysis (see, for example, refs 3,5-7)? and so on. Certainly, there are no shortage of challenges for structural biologists with an interest in G-protein-mediated signal transduction.

1. Spickofsky, N. et al. Nature struct. Biol. 1, 771-780 (1994).

2. Clapham, D.E. \& Neer, E.J. Nature 365, 403-406 (1993).

3. Conklin, B.R. \& Bourne, H.R. Cell 73, 631-641 (1993)

4. Artemyve, N.O. \& Hamm, H.E. Nature struct. Biol. 1, 752-754 (1994). 5. Noel, I.P., Hamm, H.E. \& Sigler, P.B. Nature 366, 654-662 (1993).

6. Lambright, D.G., Noel, J.P., Hamm, H.E. \& Sigler, P.B. Nature $369,621-628$ (1994).

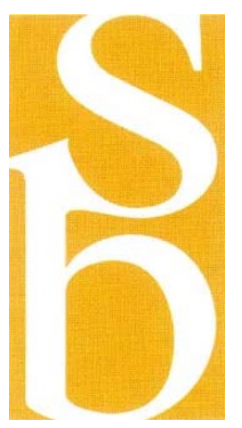

